\title{
Is It Possible to Have Neuromodulation Therapies to Treat Insomnia through Circadian Process Activation?
}

\author{
Heon-Jeong Lee ${ }^{1,2}$ \\ Editor-in-Chief \\ 'Department of Psychiatry, Korea University College of Medicine, Seoul, Korea \\ ${ }^{2}$ Chronobiology Institute, Korea University, Seoul, Korea
}

Insomnia is a common sleep disorder. It is currently defined as subjective difficulty falling asleep, staying asleep, or inability to fall asleep after waking up too early. Hypnotic medications are a popular short-term treatment, as they provide immediate subjective relief during periods of high stress, even when there may be little or no objective benefit. However, hypnotics are not a good choice for the long-term treatment of insomnia because of tolerance, dependency, and numerous medical and psychologic risks. Cognitive behavioral therapy for insomnia (CBT-I) is effective for chronic insomnia and is usually recommended as the first-line treatment. CBT-I is a structured program that helps people identify and replace negative thoughts and behaviors that cause or worsen their sleep problems. CBT-I has the merit of no dependency and little other risk apart from sleepiness early in treatment. CBT requires much time and effort to make it effective. Some CBT benefits can be gained from internet treatment programs and written materials, but there is considerable expense when a therapist is needed for adequate effectiveness. Therefore, insomnia is still considered difficult to overcome.

Insomnia causes not only sleep loss but also hyperarousal during both night and day. Sleep is regulated by a complex interaction between the homeostatic process (a drive to sleep, which builds up during wakefulness) and the circadian process (an independent 24-hour sleep-wake oscillatory rhythm that modulates sleep propensity). As poor sleep may result from either or both of these two active processes in the brain, insomnia treatment with neuromodulation should target both processes.

In this issue of Chronobiology in Medicine, two review articles have been published on neuromodulation modalities that may be used for the treatment of insomnia in the future. Yoon [1] reviewed transcutaneous auricular vagus nerve stimulation (taVNS) therapy for insomnia. As the vagus nerve is involved in maintain- ing homeostasis, stimulating it has long attracted the attention of investigators. Although VNS was approved by the United States' (US) Food and Drug Administration (FDA) for managing treatment-refractory epilepsy in 1997 and for treatment-resistant depression in 2005, its application in clinical practice has been difficult because of the high cost and need for surgery, as well as limited benefits. Therefore, to overcome these limitations, non-invasive taVNS was developed.

Jo et al. [2] introduced low-intensity transcranial focused ultrasound stimulation (tFUS) technology as a potential treatment modality for insomnia. Among various direct brain stimulation techniques, ultrasound has been proposed for neuromodulation owing to its distinct advantages of high spatial resolution and in-depth targeting and has been proven safe in humans. US FDA approved tFUS to treat essential tremor and tremor-dominant Parkinson's disease. Several clinical trials of tFUS are currently underway for various neuropsychiatric disorders, such as depression, obsessive compulsive disorders, anorexia nervosa, and Alzheimer's disease. A new miniaturized device based on microelectromechanical systems (MEMS) technology has been introduced for neuromodulation. The authors summarized the new types of MEMS-based tools of tFUS that are needed to understand the mechanisms of tFUS and develop an effective treatment modality for brain disorders.

In developing neuromodulation therapies, circadian rhythms, being an integral process of sleep physiology, should be emphasized. People in modern societies have difficulty maintaining a sound circadian rhythm because of indoor lifestyles, insufficient or ill-timed daytime lighting, or artificial lighting at night. There are evidences that circadian factors are important in insomnia and depression [3]. Disruption of the circadian rhythms can cause insomnia, and any brain treatment method will vary in efficacy depending on the time of day it is given. New neuromodulation mo- 
dalities should be developed in consideration of the circadian process as well as the homeostatic process.

Acknowledgments

This study was supported by the Korea Health 21 R\&D Project funded by the National Research Foundation of Korea (2017M3

A9F1031220).

\section{ORCID iD}

Heon-Jeong Le (D)

https://orcid.org/0000-0002-9560-2383

\section{REFERENCES}

1. Yoon HK. Therapeutic application of transcutaneous auricular vagus nerve stimulation in primary insomnia. Chronobiol Med 2019;1:51-54.

2. Jo Y, Oh C, Lee HJ. Microelectromechanical systems-based neurotools for non-invasive ultrasound brain stimulation. Chronobiol Med 2019; 1:55-59.

3. Kripke DF. When our body clocks run late: does it make us depressed? Ann Transl Med 2016;4:178. 